\title{
Neutrophil pyroptosis: new perspectives on sepsis
}

\author{
Lu Liu ${ }^{1} \cdot$ Bingwei Sun ${ }^{1}$
}

Received: 28 August 2018 / Revised: 6 March 2019 / Accepted: 7 March 2019 / Published online: 14 March 2019

(c) Springer Nature Switzerland AG 2019

\begin{abstract}
Pyroptosis is a caspase-1 or caspase-4/5/11-dependent programmed cell death associated with inflammation, which is initiated by inflammasomes or cytosolic LPS in innate immunity. Sepsis is a life-threatening organ dysfunction caused by an imbalance in the body's response to infection. It is a complex interaction between the pathogen and the host's immune system. Neutrophils play the role of a double-edged sword in sepsis, and a number of studies have previously shown that regulation of neutrophils is the most crucial part of sepsis treatment. Pyroptosis is one of the important forms for neutrophils to function, which is increasingly understood as a host active immune response. There is ample evidence that neutrophil pyroptosis may play an important role in sepsis. In recent years, a breakthrough in pyroptosis research has revealed the main mechanism of pyroptosis. However, the potential value of neutrophil pyroptosis in the treatment of sepsis did not draw enough attention. A literature review was performed on the main mechanism of pyroptosis in sepsis and the potential value of neutrophils pyroptosis in sepsis, which may be suitable targets for sepsis treatment in future.
\end{abstract}

Keywords Pyroptosis · Neutrophil · Sepsis · Caspase $\cdot$ Inflammasome $\cdot$ IL-1 $\cdot$ IL-18 $\cdot$ PITs

\section{Introduction}

Pyroptosis is a programmed cell death process associated with inflammation. It is characterized by apoptosis and necrosis in morphology $[1,2]$ (Table 1). Cells that undergo pyroptosis will form pyroptotic bodies similar in size to apoptotic bodies, with pyknosis and chromatin damage [3]. Large numbers of pores formed on the cell membrane causes it to lose integrity. Eventually, the membrane lysis arises, and the intracellular content is released to induce inflammation. In apoptotic cells, eflux of potassium and chlorine resulting in cell contraction is often observed, while necrosis is a selective ion overload that causes water influx and then cells to swell and die [4]. When the cell undergoes pyroptosis, the nonselective pores of the GSDMD formed on the cell membrane lose the natural ion gradient, which may be due to a slight swelling of the cell caused by the intracellular nonionic penetrant driving water into the cytoplasm $[3,5]$, and there are reports revealing that this swelling can

Bingwei Sun

sunbinwe@hotmail.com

1 Department of Burns and Plastic Surgery, Affiliated Hospital, Jiangsu University, 438 Jiefang Rd.,

Zhenjiang 212001, Jiangsu, China be blocked by extracellular osmoprotectant or glycine [6]. A recent report suggested that cell death and membrane lysis are uncoupled, inhibiting cell membrane lysis but not preventing cell death [7]. Compared to pyroptosis, necrosis is more like a process of cell explosion while pyroptosis undergoes cytoplasm flattening caused by plasma membrane leakage $[3,8]$.

Pyroptosis is first detected in macrophages and its related diseases [9], and then a large number of reports confirmed that pyroptosis may also occur to neutrophils [24, 25]. The research on neutrophil pyroptosis has attracted more and more attention in recent years, but the role of neutrophil pyroptosis in sepsis still did not cause enough attention. As we all know, sepsis is a life-threatening organ dysfunction caused by a dysregulated host response to infection [26]. It is an imbalance between the pro-inflammatory and antiinflammatory mediators. However, early sepsis mortality is caused by an acute, deleterious pro-inflammatory response. Neutrophils are the most abundant natural immune cells in a human body that play paradoxical roles in the progression of sepsis. In the early stage of sepsis, neutrophils first arrive at the site of infection [27], secrete important cytokines and chemokines, and obliterate pathogenic microorganisms by phagocytosis, degranulation, and release of ROS and Nets $[28,29]$. And in severe sepsis, the release of various 
Table 1 Comparison of pyroptosis, apoptosis, necrosis and NETosis

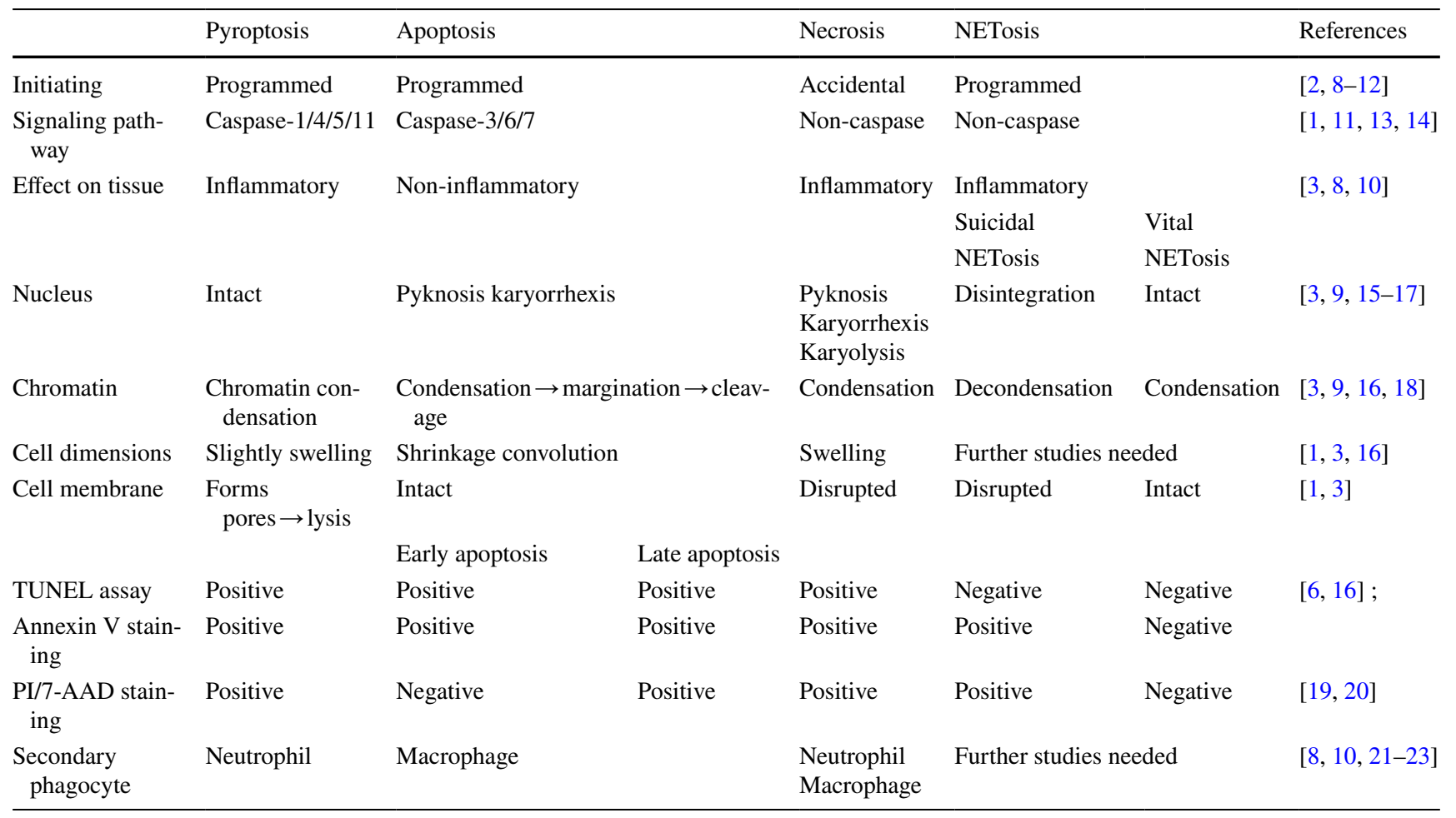

enzymes, inflammatory mediators in heart, lung, kidney and other vital organs by a large number of activated neutrophils, but with chemotactic dysfunctions, leads to tissue cell damage, and ultimately to the development of multiple organs function failure [30]. Some evidences suggest that the pyroptosis of neutrophils is an important way for neutrophils to function during sepsis and that neutrophils continuously synthesize and secrete IL- $1 \beta$ and IL-18 during pyroptosis. Eventually, neutrophils swell slightly and develop a membrane lysis, producing cytoplasmic DAMP (damage-associated molecular patterns) [10] and releasing a large number of immunomodulatory cytokines such as IL-10, IL-13, chemokines such as IL-8, MIP-1 $\alpha$, and myeloperoxidase (MPO), cathepsin $\mathrm{G}$ and other granzymes [31]. After the neutrophil membrane lysis, intracellular pathogens form a pore-induced intracellar trap (PIT) though they have not been released directly extracellularly. Via the complement and the scavenger receptor to coordinate the innate immune response, PITs promote the recruitment of neutrophils to release ROS or secondary phagocytosis to kill pathogens $[21,22]$. Under the combined effects of these cytokines and structures, neutrophil pyroptosis plays the role of inflammatory signal amplifiers in the recruitment of immune cells, There is evidence that neutrophil pyroptosis is beneficial to bacterial clearance during infection [21] and that neutrophils pyroptosis is even more essential for certain types of pathogen infections such as Salmonella infection [32]. However, because the neutrophils have been proved to be the main source of IL-1 $\beta$ in the infection $[24,25]$, excessive neutrophil pyroptosis is obviously harmful in the early hyperinflammatory state in sepsis. Numberous studies have confirmed that Caspase-1/11 knockout and IL-1 $\beta / \mathrm{IL}-18$ knockout can improve the survival rate in CLP mice model and septic shock mice model [33-35].However, another septic shock model shows that knocking out IL-1beta and IL-18 is useless [36]. These seemingly contradictory conclusions suggest that we should have different regulations on neutrophil pyroptosis in different stages of sepsis and different types of bacterial infection. Therefore, the study and summarization of the main mechanism of pyroptosis in sepsis and the potential value of neutrophil pyroptosis in sepsis is of great significance for the treatment of sepsis.

\section{Neutrophils in sepsis}

In the early phase of sepsis, neutrophils are essential for pathogen control. Studies on neutrophils obtained that most neutrophils normally undergo apoptosis within $24 \mathrm{~h}$. Interestingly, due to the increased release of immature neutrophils and the delayed apoptosis of circulating neutrophils, a large number of circulating neutrophils of various degrees of maturation can be detected in sepsis patients. Investigation of sepsis patients and animal models of sepsis revealed disrupted neutrophil functions, including impaired neutrophil migration, impaired clearance of bacteria, reduced 
production of reactive oxygen species (ROS) and disordered release of cytokines. [37] Recent studies have shown that some subsets of neutrophils in sepsis patients can secrete a large amount of IL-10, which is an immunosuppressive cytokine that can suppress the proliferation of $\mathrm{T}$ lymphocyte [38]. Other evidences show complex interactions between the neutrophils and complement system, which cause complement-induced innate immune damage during sepsis. This review focuses on another important way in which neutrophils regulate immunity in sepsis: pyroptosis.

Pyroptosis is a programmed cell death process dependent on caspase- 1 and -11 , considered to be an immune response involved in the acute bacterial and viral infections, as well as the exposure of bacterial toxins and intracellular pathogens eradication [14] (Fig. 1). Caspases, a class of cysteine proteases widely expressed in mammals, are mainly associated with programmed cell death and inflammatory responses, some of which play a key role in antiviral immunity. Among them, Caspase-1, Caspase-4, Caspase-5 and Caspase- 11 have proved to dominate the process of pyroptosis, and the role of Caspase-13 and Caspase-14 in pyroptosis is still controversial. Caspase-4, Caspase-5 and Caspase-11 are homologous proteins, Caspase- 4 and Caspase- 5 are expressed in mice and Caspase-11 is expressed in human [14, 39]. When pathogens exist, natural immune cells, including neutrophils, macrophages, dendritic cells and other immune participating cells such as epithelial cells, endothelial cells and fibroblasts, detect microorganisms by identifying, via pattern recognition receptors (PRRs), highly conserved pathogenassociated molecular patterns (PAMPs), including flagellin, peptidoglycan (PGN) and lipopolysaccharide (LPS). Other evidences reveal that PRRs are also responsible for identifying endogenous molecules released from damaged cells, called danger-associated molecular patterns (DAMPs) [40]. The activation of innate immunity depends on the recognition of both PAMPs and DAMPs, which is beneficial to the immune system's tolerance to commensal probiotics and non-pathogenic bacteria [41]. At the same time, there is a growing number of evidence that DAMPs is essentially cytotoxic and not only depends on the involvement of inflammasomes in this process [42]. The PRR family consists of three categories: TLRs, NLRs and RLRs. Some NLRs can form a complex with specific functions called inflammasome, including NLRP1, NLRP3, NLRC4 and AIM2 involved in signal recognition during pyroptosis. NLRP1, NLRP3 and NLRC4 mRNAs were expressed in both human and mouse neutrophils at similar or greater levels than other cell types whose function is well studied, such as marrow-derived macrophages (BMDMs) or bone marrow-derived dendritic cells (BMDCs) [32]. The NLR family is characterized by the presence of a central nucleotide-binding and oligomerization domain (NACHT), the repeat domain of caspase recruitment (LRRs) and N-terminal (CARD) or PYRIN domain (PYD)
[43-45]. GSDMD (gasdermin D), a member of the gasdermin protein family, is essential for pyroptosis in humans and mice. The structure of GSDMD has both the $\mathrm{N}$ terminal domain and the $\mathrm{C}$ terminal domain. Studies have shown that its $\mathrm{N}$ terminal domain plays a major role in inducing pyroptosis. The first loop on GSDMD-C that inserts into the N-terminal domain (GSDMD-N) helps stabilize the conformation of the full-length GSDMD [46], whereas the over expressed C end blocks GSDMD-N dependent pyroptosis [5, 47, 48].

\section{Canonical inflammasome induced pyroptosis}

In bacterial infection, pattern recognition receptors (NLRP1b, NLRP3, NLRC4) recognize microbial PAMPs, and then, via ASC (apoptosis-associated speck-like protein adaptor protein containing a CARD) indirectly connected with procaspase-1, form a Caspase-1-dependent inflammasome. ASC docks onto the inflammasome hub via pyrin-pyrin interactions and then recruits Caspase-1 via CARD-CARD interactions. However, ASC can also interact with itself, recruiting additional ASC molecules via pyrin-pyrin and CARD-CARD interactions, where all of the ASC within a cell is recruited via the cascade effect to a single subcellular location, which has been called the 'ASC focus' or 'ASC speck'. In the ASC focus, the pro-caspase- 1 in the form of the dimer is cleaved into P10 and P20 subunits to form catalytically active caspase-1, where pro-IL- $1 \beta$ is activated into mature IL-1 $\beta$ [15]. In addition, NLRP1 and NLRC4 can also directly connect and activate Caspase-1 without depending on ASC (Fig. 2). Different inflammasomes detect different intracellular contamination and perturbation: (a) Gram negative bacteria such as $S$. typhimurium [32], S. flexneri [49] and P. aeruginosa [50] or L. pneumophila [51] and Brucella [52] can transport, respectively, through Type III secretion system or through Type IV secretion system, the flagellin into the neutrophil cytoplasm, which are to be recognized by NLRC4 inflammasome. (b) Exotoxins secreted by Gram-positive bacteria and some Gram-negative bacteria can enter the cytoplasm by ionophore, pore former [53], protease [54] and other mechanisms, which are to be recognized by NLRP1 or NLRP3 inflammasome. (c) Other PAMPs, DAMPs and cytosolic low $\mathrm{K}^{+}$can be recognized by NLRP3 inflammasome. These inflammasomes recruit pro-caspase -1 via ASC and activate Caspase-1 ultimately [11, 15, 55]. The activated Caspase- 1 cleaves the connecting part between $\mathrm{N}$-terminal and C-terminal of GSDMD rapidly, removes the inhibitory effect of $\mathrm{C}$-terminal on the $\mathrm{N}$-terminal and releases the GSDMD N-terminal to connect with the phosphoinositide of cell membrane and generate the oligomerization. Then the formation of pores disrupts the osmotic balance of the cell membrane, leading to cell swelling and 


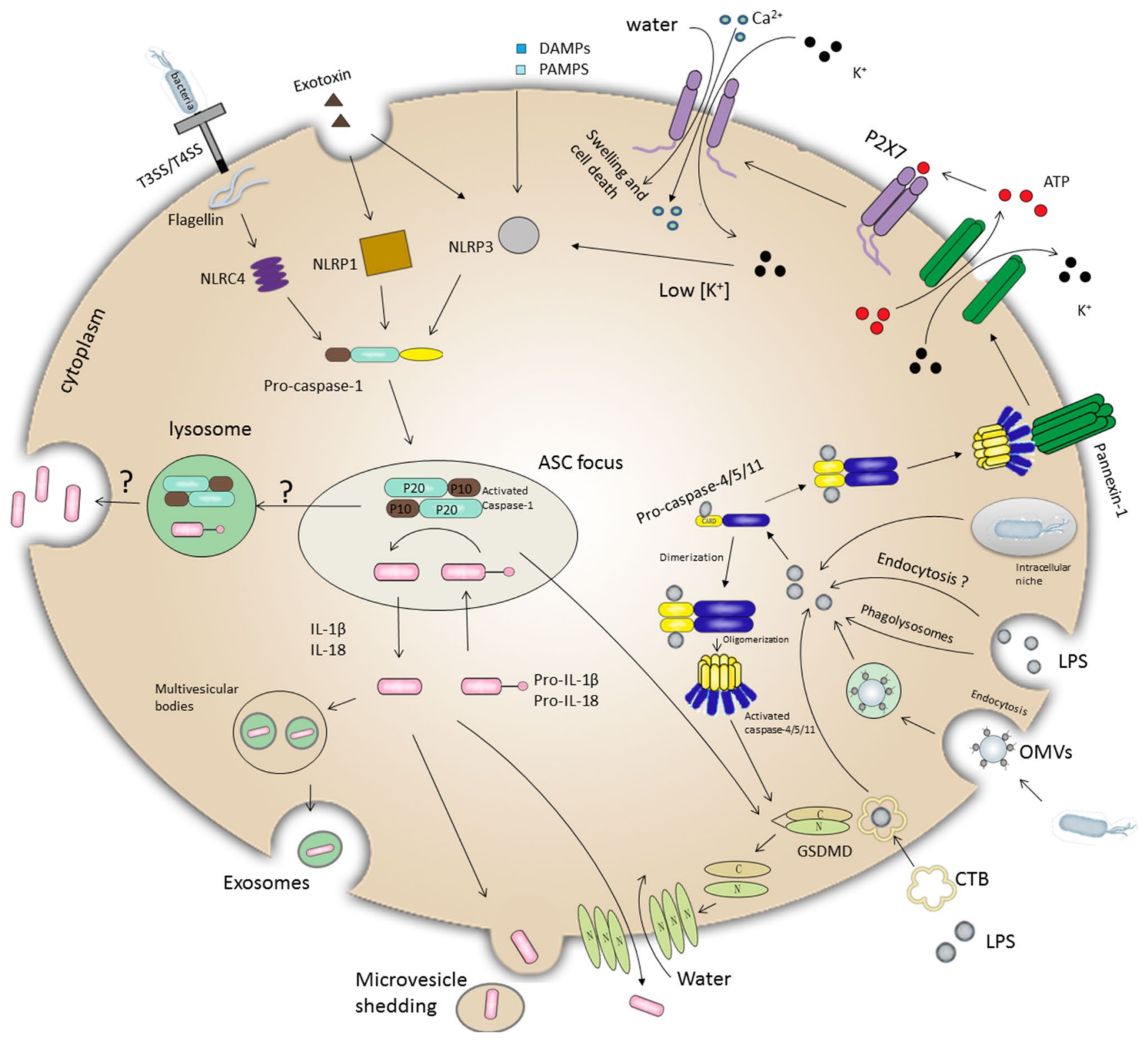

Fig. 1 In sepsis, there exist the neutrophil recognition of flagellin, exotoxins, other PAMPs and DAMPs by caspase- 1 and the recognition of cytosolic LPS by caspase 4/5/11. In this process, NLRC4 is responsible for the detection of bacterial flagellin; NLRP1 is responsible for the detection of deadly toxins, and NLRP3 is responsible for the detection of other PAMPs and DAMPs as well as the cytoplasmic low potassium induced by ATP-mediated P2X7 signaling. Activation of caspase- 1 and production of mature IL-1, beta and IL-18 are carried out in ASC focus. The pro-caspase-1 in the form of the dimer is cleaved into P10 and P20 subunits to form catalytically active caspase-1, and LPS triggers Caspase-11 oligomerization and activates its proteolytic activity. Caspases of the two active states mentioned above cleave the GSDMD and generate the oligomerization to form pores on the cell membrane, eventually leading to membrane lysis and pyroptosis. Other reports suggest that Caspase- 11 induces pyroptosis via ATP-mediated $\mathrm{P} 2 \mathrm{X} 7$ signaling, resulting in cytoplasmic low $\mathrm{K}^{+}$activation of NLRP3 inflammasome and Caspase-1 induced IL-1 beta and IL-18 maturation and release. There are probably five ways of extracellular LPS entering cytoplasm: (a) through an unknown endocytosis. (b) Gram-negative bacteria in the intracellular vacuoles release LPS directly into the host cytoplasm by IFN- $\gamma$ induced GTPase action. (c) Gram-negative bacteria secrete outer membrane vesicles (OMV) wrapped LPS that enters the cytoplasm via endocytosis. (d) Extracellular LPS enters the cytoplasm via the carrier of CTB. (e) HMGB1 destabilizes phagolysosomes for the transfer of LPS to cytosolic caspase-11. In neutrophils and other cells, three possible different ways have been found for mature IL- $1 \beta$ and IL-18 to release extracellularly: (a) mature IL-1 $\beta$ and IL-18 release via extracellularly through the membrane pores. (b) Monocyte encapsulates the activated caspase- 1 and cytokine substrates into lysosomes and releases the processed cytokines via the lysosome to the cell surface. (c) Dendritic cells, microglia and macrophages can release the cytokinecontaining vesicles after ATP stimulates Caspase-1. There are two different ways of release here: the release of cell surface fusions of multivesicular bodies and the release of microbubbles directly from the cell membrane 


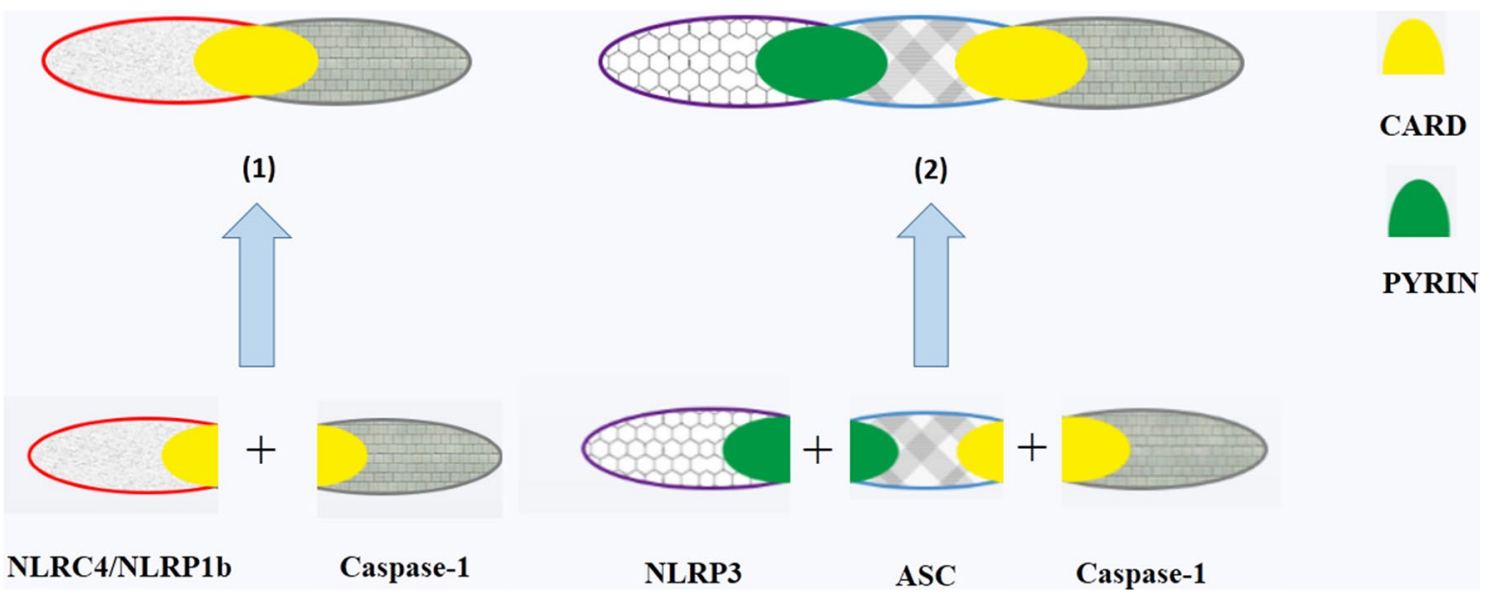

Fig. 2 (1) NLRC4 and mouse NLRP1b contain the CARD domain that binds directly to the CARD domain of Caspase- 1 to trigger Caspase-1. (2) The ASC contains the Pyrin signal domain and the CARD

membrane lysis, and releasing large amounts of cell contents causing inflammation $[5,47,48]$. Interestingly, activation of inflammasomes does not imply that the cells are destined to lyse and that certain toxins, such as melittin, can prevent cell lysis but induce NLRP3 inflammasome activation and IL-1 $\beta$ release [56]. In some cases, such as acute Salmonella infection, NLRC4 inflammasome sustained activation of neutrophils to release IL-1 $\beta$ without pyroptosis [32]. There are still controversies in previous reports on the pore diameters formed on the membrane of GSDMD, such as $15 \mathrm{~nm}$ inner and $32 \mathrm{~nm}$ outer [47], 12-14 nm [5], $13 \mathrm{~nm}$ [57], $20 \mathrm{~nm}$ [58] and 1.1-2.4 nm [6], respectively. Caspase-1 activated GSDMD can promote proIL- $1 \beta$ and proIL-18 maturation, and the continuous production of active IL- $1 \beta$ and IL-18 $[11,15]$, which are to be released extracellularly through the membrane pores formed by GSDMD or other ways [59, 60]. In addition, studies have shown that activated GSDMD not only induces the host cell membrane to form pores, but also form pores on the surface of the bacteria infecting the host to kill the bacteria. The GSDMD N-terminal released after lysis can also kill bacteria directly outside the host, including Escherichia coli, Staphylococcus aureus and Listeria monocytogenes [47].

\section{Non-canonical inflammasome induced pyroptosis}

Lipopolysaccharide is a major component of the cell wall of gram-negative bacteria. It can not only induce inflammation $[61,68]$ and autophagy [62], but can also induce cell pyroptosis $[13,63-65]$. Under the stimulation of intracellular LPS, Caspase-11 can specifically bind to the lipid A of LPS, which triggers Caspase-11 oligomerization, domain, NLRP3 that contains the Pyrin signal domain can be combined with ASC's Pyrin signal structure and the CARD domain of ASC can connect with the CARD domain of Caspase-1

activates its proteolytic activity and cleaves the GSDMD to form a large number of pores on the cell membrane, eventually leading to membrane lysis and pyroptosis $[13,63$, 64]. Several reports also reveal that the binding of intracellular LPS to Caspase-11 triggers Caspase-11 oligomerization, and the activated Caspase-11 can cleave Pannexin-1 to make it open [65]. Since intracellular ATP concentration is one million times extracellular ATP concentration [66], the intracellular ATP released through the Pannexin-1 channel to the extracellular activation of $\mathrm{P} 2 \mathrm{X} 7$ receptor leads to P2X7 channel opening, rapidly triggers cytoskeleton destruction and membrane PS flip, blebbing, microvesicle shedding and eventually leads to the destruction and lysis of the cell membrane [67]. P2X7 receptor activation mediates membrane blebbing in at least two different ways: calciumdependent mechanisms and calcium-independent RhoA and ROCK-1 mechanisms [68]. In the pannexin-1 channel to open the release of ATP, under the concentration gradient of $\mathrm{K}^{+}$inside and outside, $\mathrm{K}^{+}$is released in large quantities extracellularly, resulting in cytoplasmic low $\mathrm{K}^{+}$activation of NLRP3 inflammasome and inducing pyroptosis via the ASC activation of Caspase- 1 to release mature IL- $1 \beta$ and IL-18 [69-71]. Other reports reveal that not only can the Pannexin- 1 channel release $\mathrm{K}^{+}$to the extracellular matrix, the $\mathrm{P} 2 \mathrm{X} 7$ receptor also has this ability, and the latter plays a major role in the process of intracellular low potassium activation of NLRP3 [71, 72]. Previous reports indicate that the expression and function of $\mathrm{P} 2 \mathrm{X} 7$ receptors on human and mice neutrophils are contradictory $[70,73]$ or $\mathrm{P} 2 \mathrm{X} 7$ is present in the cytoplasm rather than in the human neutrophil surface [74]. However, recent reports have demonstrated that P2X7 receptors are widely expressed on human and mice neutrophils and exhibit a certain racial differences [70, 71]. 


\section{The source of cytosolic LPS in pyroptosis}

In severe sepsis, a great deal of LPS is released into the blood circulation, activating a large number of neutrophils via TLR2 and TLR4 receptor. At the same time, LPS inhibits neutrophil chemotaxis through autocrine ATP signaling pathway [75], resulting in extensive damage to tissues and organs [30]. At present, the source of cytosolic LPS is still not entirely clear, and obviously the great deal of extracellular LPS does not directly penetrate through the membrane into the cytoplasm during sepsis. It may be that the extracellular LPS enters cytoplasm via a special transport way or through the channel generated during pyroptosis, or it may originate from the release of bacteria in the inner niche under specific conditions. Neutrophils are a key member of the immune system with a strong phagocytic capacity, so neutrophils in the sepsis can swallow the pathogen to form phagocytosis to kill the pathogen, where LPS cannot be released into the cytoplasm [76]. Many microbes have been found to survive in neutrophils, such as Salmonella [77], Neisseria gonorrhoeae [59, 78], S. aureus [79], Chlamydia pneumoniae [80], Burkholderia pseudomallei [60], Anaplasma phagocytophilum [81] and L. monocytogenes [82]. Another report suggests that the survival of Gramnegative bacteria in the intracellular vacuoles can directly release LPS into the host cytoplasm, which requires IFN- $\gamma$ to induce GTPase action to cause bacteria to escape from the vacuole or to break the vacuole [83]. Several reports suggest that ATP, which is abundant in inflammation sites, activates the P2X7-dependent Pannexin-1 half channel pore formation and may allow extracellular LPS to promote the LPS entry into the cytoplasm via either Pannexin-1 channels or Pannexin-1 mediated indirect effects [44, 84]. P2X7 is a selective cation channel in the presence of ATP stimulation, and when the divalent cation level is low, the cationic channel can be converted into small pores and ions that can penetrate up to $900 \mathrm{Da}$ [73]., Because the LPS molecular weight is about 5-15 kD, and Pannexin- 1 channel can penetrate about $1 \mathrm{kD}$ [85], it is obvious that LPS cannot directly penetrate through the Pannexin-1 or P2X7 channel. According to a previously reported LPS electroporation model, the extracellular LPS that gets free access to the cytoplasm via the membrane pore can cause the vast majority of cells to undergo pyroptosis [86]. As stated above, the extracellular LPS stimulation of neutrophils can also activate TLR4P38-Cx43 pathway to autocrine ATP extracellularly [75]. Therefore, the existence and efficiency of this mechanism where the LPS directly or indirectly enters the cytoplasm via the Pannexin- 1 channel remains to be further studied and discussed.

The other two distinct extracellular LPS transport mechanisms require a specific vector. The first is cholera toxin B (CTB), which can be used as a carrier binding LPS and transported to cytoplasmic induction of non-canonical inflammasome pyroptosis [87-89], and this combination is required for LPS type. There are data showing that only LPS O111:B4 can be combined with CTB to induce noncanonical inflammasome pyroptosis, but LPS O55: B5 and LPS O127: B8 have no such ability [87]. Another mechanism reported in recent years is Gram-negative bacteria secrete outer membrane vesicles (OMV) wrapped LPS that enter the cytoplasm via endocytosis. OMVs are vesicles between 20 and $250 \mathrm{~nm}$ produced in a programmatic way; they are not by-products of bacterial cell wall damage or bacterial dissolution. Bacteria that poorly produce OMVs elicit low-level pyroptosis and IL-1 maturation [63]. OMVs can carry LPS, phospholipids, peptidoglycan, outer membrane proteins (OMPs), cell wall components, proteins (periplasmic, cytoplasmic, and membrane-bound), ion metabolites and signaling molecules [90], and many bacterial OMVs also carry nucleic acids (DNA, RNA) [91]. Following the clathrin-mediated endocytic uptake, OMVs transmit LPS into the cytosol from early endocytic compartments and eventually activate Caspase-11 to trigger pyroptosis [63]. Early endosomal escape allows OMV-bound LPS to reach cytosol functionally intact and avoid complete degradation in the lysosomes. There is an evidence that OMVs can strongly stimulate neutrophils to secrete IL-1 $\beta$. Therefore, the neutrophil pyroptosis caused by cytosolic LPS is very likely transport by OMVS too. A recent report confirms that HMGB1 destabilizes phagolysosomes for the transfer of LPS to cytosolic caspase-11 [92]. The author's ample evidence in the article seems to have brought us closer to solving the mystery.

\section{Roles and release mechanisms of IL-1 $\beta$ and IL-18 in pyroptosis}

The interleukin-1 (IL-1) cytokine family, an important regulator of innate immunity and adaptive immunity, plays an important role in the host's defense against infection and inflammatory injury. The family includes IL-1, IL-18 and IL-33 and the recently found IL-36 and IL-37, in which both IL-1 $\beta$ and IL-18 mediate the inflammatory response [93, 94]. IL- $1 \beta$ and IL-18 have been shown to be the most important cytokines during pyroptosis $[69,95]$. IL- $1 \beta$ is usually produced by tissue macrophages, DC cells, blood mononuclear cells, neutrophils, B lymphocytes and NK cells, but generally not by fibroblasts and epithelial cells [94]. In the early stage of sepsis, the resident macrophages in the site of infection secrete IL-1 $\beta$ to recruit neutrophils. Then the partial neutrophils to the site of infection undergo pyroptosis and become the main source of IL-1 beta secretion, mediated by the positive signal amplification circuit to recruit 
more neutrophils to the infection sites [32]. In the meantime, one report showed that neutrophils were the main source of IL-1 $\beta$ secretion in bone marrow during infection [24] and another reported that neutrophils were the main source of IL-1 $\beta$ and IL-18 in BALF during infection [25].Therefore, we can assume that neutrophil pyroptosis in sepsis controls the level of IL-1 $\beta$ and IL-18. Another important cytokine, $\mathrm{IL}-18$, can be detected in many cells including Kuppfer cells, monocytes, dendritic cells (DCs), macrophages, keratinocytes, chondrocytes, intestinal epithelial cells, Synovial fibroblasts and osteoblasts [96-98]. In a case of sepsis, LPS first stimulates the production of pro-IL-1 $\beta$ and Pro-IL-18 by TLR or RLR. Then, NLR mediates inflammasome activation and facilitates post-translational processing, which is necessary for its secretion and biological activity [99]. IL-1 $\beta$ is one of the most potent proinflammatory cytokines known at present. It has an obviously protective effect in acute infection, including rapid recruitment of neutrophils to inflammatory sites, activation of endothelial adhesion molecules, induction of cytokines and chemokines, induced febrile reaction and stimulating specific immune responses such as Th17 response [99]. IL-1 $\beta$ has a protective effect in several bacterial, viral and fungal infection models, and the use of IL-1 receptor antagonist, IL-1R, to antagonize IL-1 increases the susceptibility to bacteria. The most special function of IL-18 is to promote Th1 cells, NK cells and cytotoxic $\mathrm{T}$ lymphocytes to produce interferon- $\gamma(\mathrm{IFN}-\gamma)$ and promote CD8+ T cells and NK cell proliferation. In addition, IL-18 can also stimulate other inflammatory cells to secrete cytokines such as tumor necrosis factor $\alpha$ (TNF- $\alpha)$, IL-1 $\beta$, IL-8 and GM-CSF [100].

The mechanism of the secretion of IL- $1 \beta$ and IL-18 is also controversial in pyroptosis. In addition to the release of IL- $1 \beta$ and IL-18 via extracellularly through the membrane pores, there are two more possible release mechanisms that have been found in neutrophils and other cells: (a) monocyte encapsulates the activated caspase- 1 and cytokine substrates into lysosomes and releases the processed cytokines via the lysosome to the cell surface [101]. Although this mechanism of secretion can avoid pyroptosis and continue to secrete cytokines, it has been considered as not dominant [102]. (b) Dendritic cells, microglia and macrophages can release the cytokine-containing vesicles after ATP stimulates Caspase-1 $[103,104]$. There are two different ways of release here: the release of cell surface fusions of multivesicular bodies and the release of microbubbles directly from the cell membrane [104-106]. The specific mechanism for the release of IL- $1 \beta$ and IL-18 in the case of neutrophil pyroptosis is still controversial because of the short life of neutrophils and they cannot be proliferate, it is difficult to study the release of granules and cytokines. Direct release of IL-1 $\beta$ and IL-18 after cell membrane dissolution is present. However, the continuous maturation of IL- $1 \beta$ and mature IL-18 release require the continuous activation of Caspase-1, so this way is obviously not the major one. A recent article that attracted wide attention showed that the macrophage IL- $1 \beta$ was released actively through the holes formed by GSDMD [107], but it has not been proved directly in neutrophils and require further study.

\section{Pore-induced intracellular traps triggered by pyroptosis}

Caspase- 4 and Caspase-4/5/11 trigger the pathway of pyroptosis, eventually causing cell membrane lysis, that is the release of intracellular contents and cytokine, which is an important natural immune response. Recent studies have shown that natural immune cells such as macrophages and neutrophils have shown other specific ways to function in pyroptosis: the formation of PITs, rather than the mere release of intracellular pathogens and contents to the extracellular environment. These reports reveal that organelles and cytoskeleton do not have mere separation and diffusion after the membrane lysis, but remain in the corpse of the cells to form an intracellular trap-like structure to restrict surviving pathogens, which is a structure called poreinduced intracellular traps(PITs) [21, 22]. Then, the pathogens trapped in PITs were subsequently killed by neutrophil ROS or by secondary phagocytosis of other neutrophils [10]. PITs are conceptually similar to Nets, both by preventing bacteria from spreading and promoting clearance. The difference between the two is that Nets are directed against extracellular bacteria, while PITs are targeted to intracellular niche. Nets and PITs are independent of each other. In Netsdeficient mice (MPO -/- and Elane -/-) the formation of PITs was not affected [21]. Nets are essentially bactericidal because they contain antibacterial peptides and enzymes and produce ROS [108-112], while PITs damage, but do not kill bacteria. After the formation of PITs, the killing or secondary phagocytosis of neutrophils ROS apparently poses a question: how does neutrophil discover PITs?

Apoptosis or necrotic cells will release the "Find-me" signal, expose the "Eat-me" signals and lose "Don't eat me" signals, which combine to promote the clearance of apoptotic and necrotic cells [23, 113]. When PIT is formed, "Find-me" signals, such as microbial surface C5a or chemokines and LPC released from pyroptosis cells, are first released. Then the various "Eat-me signals" such as PS and DNA are rapidly exposed, while several "Don't eat me" signals such as CD47, CD31 are lost or modified. Then together with some extra elements of expression, they eventually reach a new balance between "Eat-me" signals and "Don't eat me "signals, causing neutrophils to eventually reach the environs of PITs to undergo efferocytosis and destroy the pathogens entrapped inside by a secondary killing $[114,115]$. After 
the membrane lysis of phagocytic cells, PITs are bound to form, and PITs are small enough to retain organelles and bacteria [21].The clearance of pyroptotic neutrophils is actually equal to the clearance of PITs and it seems to induce a pro-inflammatory reaction.

\section{Conclusions and perspectives}

Pyroptosis has been shown to be an important natural immune response. Although a variety of classical and nonclassical inflammasome-induced pyroptosis have been described, there are still many controversies concerning the specific details of pyroptosis. With the research of pyroptosis deepening gradually, the possibility of pyroptosis applied to sepsis treatment is adequately amplified. Many studies have confirmed that Caspase-1/11 knockout and IL-1ß/IL-18 knockout can improve the survival rate in CLP and septic shock mice model (Table 2), but clinical trials failed [116]. It seems to confirm that the inhibition of IL-1 and IL18 are harmful. However, due to the pro-inflammatory and antiinflammatory responses in sepsis, the same treatment may lead to different outcomes for patients at different stages of sepsis. Furthermore, differences in sepsis-causing bacteria may also affect outcomes.

On the basis of preclinical studies, some clinical trials have been carried out. Most of these clinical trials focused on IL- $1 \beta$, the downstream of pyroptosis. However, attempts to improve sepsis survival by blocking IL-1 $\beta$, although effective against animal models [36], failed in some previous human trials $[121,122]$. Encouragingly, a recent III phase clinical trial supports the possibility that anakinra (recombinant interleukin-1 receptor antagonist) treatment provides a survival benefit in septic patients with features of Macrophage Activation Syndrome [123]. A prospective trial has been initiated to validate these findings (NCT03332225; clinicaltrials.gov). Another precise clinical study shows that recombinant IL-1 receptor antagonists are beneficial to septic patients with high plasma interleukin-1 receptor antagonist (IL-1RA) [124].Since IL-1 $\beta$ induces gene expression of both itself and IL-1RA, significantly increased IL-1RA may inhibit IL-1 $\beta$, stopping the cycle of IL-1 $\beta$-IL-1RA amplification. Therefore, both subgroups of sepsis patients above actually occur to the context of cytokine storm, in which IL- $1 \beta$ plays a major role. In addition, there is another report that yielded similar results, but the sample size of sepsis patients is too small, and the results are still debatable [125]. At present, there are still few studies on the upstream molecules of pyroptosis in sepsis. A published study reveals an association of NLRP3 and Caspase-1 mRNA levels with severity of sepsis caused by cytomegalovirus (CMV) [126]. Another study suggested that caspase- 1 is a potential marker for predicting the development of sepsis after severe trauma [127]. These preclinical and clinical studies have revealed that the regulation of neutrophil pyroptosis may benefit in the treatment of sepsis. Given the important role of neutrophil pyroptosis in sepsis, the regulation of neutrophil pyroptosis may have more potential value.

Table 2 Effects of pyroptosis-related molecule-deficient mice in sepsis

\begin{tabular}{|c|c|c|c|c|}
\hline Knockout strains & Murine models & Regulation of neutrophil functions & Outcomes & References \\
\hline Caspase-1 & Septic shock (E. coli) & IL-1 $\beta$ secretion $\downarrow$ & Survival rate $\uparrow$ & [36] \\
\hline Caspase-11 & Endotoxic shock (LPS) & No information available & Survival rate $\uparrow$ & {$[117]$} \\
\hline \multirow[t]{2}{*}{ Caspase-1/11 double KO } & $\begin{array}{l}\text { Polymicrobial sepsis (Low-lethality cae- } \\
\text { cal slurry) }\end{array}$ & $\begin{array}{l}\text { Phagocytosis } \uparrow \\
\text { Transmigration } \uparrow\end{array}$ & Survival rate $\uparrow$ & [118] \\
\hline & Endotoxic shock (LPS) & No information available & Survival rate $\uparrow$ & [35] \\
\hline \multirow[t]{2}{*}{ IL-1 $\beta$} & Septic shock (E. coli) & No information available & No significant difference & [36] \\
\hline & Endotoxic shock (LPS) & No information available & Survival rate $\uparrow$ & [35] \\
\hline IL-18 & Endotoxic shock (LPS) & No information available & Survival rate $\uparrow$ & {$[10]$} \\
\hline \multirow[t]{3}{*}{ IL-1 $\beta /$ IL-18 double KO } & Septic shock $(E$. coli $)$ & No information available & No significant difference & [36] \\
\hline & Endotoxic shock (LPS) & No information available & Survival rate $\uparrow$ & {$[35]$} \\
\hline & Polymicrobial sepsis (CLP) & No information available & Survival rate $\uparrow$ & {$[35]$} \\
\hline \multirow[t]{2}{*}{ NLRP3 } & Polymicrobial sepsis (CLP) & No information available & Survival rate $\uparrow$ & [119] \\
\hline & $\begin{array}{l}\text { Polymicrobial sepsis (Low-lethality cae- } \\
\text { cal slurry) }\end{array}$ & No information available & Survival rate $\uparrow$ & [118] \\
\hline \multirow[t]{3}{*}{ ASC } & $\begin{array}{l}\text { Polymicrobial sepsis (Low-lethality cae- } \\
\text { cal slurry) }\end{array}$ & No information available & Survival rate $\uparrow$ & [118] \\
\hline & Endotoxic shock (LPS) & IL-1 $\beta$ secretion $\downarrow$ & No information available & [32] \\
\hline & Endotoxic shock (LPS) & No information available & Survival rate $\uparrow$ & [120] \\
\hline
\end{tabular}

IL-1 $\beta$, interleukin-1 beta; IL-18, interleukin-18; KO, knockout; E. coli, Escherichia coli; LPS: lipopolysaccharide; CLP, cecal ligation and puncture 
Neutrophils are the most abundant immune cells in sepsis and also the main source of IL- $1 \beta$ and IL-18. Considering the short life of neutrophils and the characteristics of its inability to proliferate, targeting the neutrophil pyroptosis specifically, which is the upstream of IL-1 and IL-18 may be better to avoid the chain reaction caused by Caspase-1/11 and IL-1 $\beta /$ IL-18 blocking. There is evidence that in the Pao1 mice lung infection model, the pyroptosis level of neutrophils in bronchoalveolar lavage fluid is less than $2 \%$ in $24 \mathrm{~h}$ [25], but contributes most of IL- $1 \beta$ production. At the same time, due to the low level of neutrophils pyroptosis, the tissue damage caused by released cytosolic contents is far from the abnormal infiltration of neutrophils in tissues and organs. All these suggest us that the great potential for the regulation of neutrophil pyroptosis in sepsis. For some types of infections such as Salmonella infection, specific regulation of neutrophil pyroptosis may bring more benefits. On the other hand, because neutrophils are relatively short-lived and cannot proliferate, the regulation of neutrophil pyroptosis seems to be easier to avoid the unpredictable risks of sepsis treatment. Therefore, the study of neutrophil pyroptosis will be a highly valuable and promising treatment for sepsis.

Acknowledgements This study was supported by the National Natural Science Foundation of China, no. 81071546, no. 81272148 and no. 81471903; by the Jiangsu Natural Science Foundation, no. BE2017695.

\section{Compliance with ethical standards}

Conflict of interest The authors declare that they have no competing interests.

\section{References}

1. Elmore S (2007) Apoptosis: a review of programmed cell death. Toxicol Pathol 35(4):495-516

2. Galluzzi L et al (2015) Essential versus accessory aspects of cell death: recommendations of the NCCD 2015. Cell Death Differ 22(1):58-73

3. Chen X et al (2016) Pyroptosis is driven by non-selective gasdermin-D pore and its morphology is different from MLKL channelmediated necroptosis. Cell Res 26(9):1007-1020

4. Dong T et al (2015) Using small molecules to dissect non-apoptotic programmed cell death: necroptosis, ferroptosis, and pyroptosis. Chembiochem A Eur J Chem Biol 16(18):2557-2561

5. Shi J, Gao W, Shao F (2016) Pyroptosis: gasdermin-mediated programmed necrotic cell death. Trends Biochem Sci 42(4):245-254

6. Fink SL, Cookson BT (2006) Caspase-1-dependent pore formation during pyroptosis leads to osmotic lysis of infected host macrophages. Cell Microbiol 8(11):1812-1825

7. Dipeso L et al (2017) Cell death and cell lysis are separable events during pyroptosis. Cell Death Discov 3:17070

8. Newton K, Manning G (2016) Necroptosis and inflammation. Annu Rev Biochem 85(1):743
9. Zychlinsky A, Prevost MC, Sansonetti PJ (1992) Shigella flexneri induces apoptosis in infected macrophages. Nature 358(6382):167-169

10. Jorgensen I, Rayamajhi M, Miao EA (2017) Programmed cell death as a defence against infection. Nat Rev Immunol 17(3):151

11. Franchi $L$ et al (2009) The inflammasome: a caspase-1-activation platform that regulates immune responses and disease pathogenesis. Nat Immunol 10(3):241-247

12. Yipp BG, Paul K (2013) NETosis: how vital is it? Blood 122(16):2784-2794

13. Yi YS (2017) Caspase-11 non-canonical inflammasome: a critical sensor of intracellular LPS in macrophage-mediated inflammatory responses. Immunology 152(2):207-217

14. Shalini $S$ et al (2015) Old, new and emerging functions of caspases. Cell Death Differ 22(4):526

15. Miao EA, Rajan JV, Aderem A (2011) Caspase-1 induced pyroptotic cell death. Immunol Rev 243(1):206-214

16. Van CS, Van DBW (2002) Morphological and biochemical aspects of apoptosis, oncosis and necrosis. Anatomia Histologia Embryologia 31(4):214-223

17. Desai J et al (2016) Matters of life and death. How neutrophils die or survive along NET release and is "NETosis" = necroptosis? Cell Mol Life Sci 73(11-12):1-9

18. Watson PR et al (2000) Salmonella enterica serovars Typhimurium and Dublin can lyse macrophages by a mechanism distinct from apoptosis. Infect Immun 68(6):3744-3747

19. Brennan MA, Cookson BT (2000) Salmonella induces macrophage death by caspase-1-dependent necrosis. Mol Microbiol 38(1):31

20. Silveira TN, Zamboni DS (2010) Pore formation triggered by Legionella spp. is an Nlrc4 inflammasome-dependent host cell response that precedes pyroptosis. Infect Immun 78(3):1403

21. Jorgensen I et al (2016) Pyroptosis triggers pore-induced intracellular traps (PITs) that capture bacteria and lead to their clearance by efferocytosis. J Exp Med 213(10):2113

22. Jorgensen I et al (2016) IL-1 , IL-18, and eicosanoids promote neutrophil recruitment to pore-induced intracellular traps following pyroptosis. Eur J Immunol 46(12):2761-2766

23. Kurosaka K et al (2003) Silent cleanup of very early apoptotic cells by macrophages. J Immunol 171(9):4672-4679

24. Mankan AK et al (2012) The NLRP3/ASC/caspase-1 axis regulates IL-1 $\beta$ processing in neutrophils. Eur J Immunol 42(3):710

25. Ryu JC et al (2017) Neutrophil pyroptosis mediates pathology of $P$. aeruginosa lung infection in the absence of the NADPH oxidase NOX2. Mucosal Immunol 10(3):757-774

26. Rhodes A et al (2017) Surviving sepsis campaign: international guidelines for management of sepsis and septic shock: 2016. Intensive Care Med 43(3):304-377

27. Kolaczkowska E, Kubes P (2013) Neutrophil recruitment and function in health and inflammation. Nat Rev Immunol 13(3):159-175

28. Bonne-Année $S$ et al (2014) Extracellular traps are associated with human and mouse neutrophil and macrophage mediated killing of larval Strongyloides stercoralis. Microbes Infect 16(6):502-511

29. Tadié J-M et al (2012) Differential activation of RAGE by HMGB 1 modulates neutrophil-associated NADPH oxidase activity and bacterial killing. Am J Physiol Cell Physiol 302(1):C249-C256

30. Fabiane $S$ et al (2016) Paradoxical roles of the neutrophil in sepsis: protective and deleterious. Front Immunol 7:155

31. Grigorieva DV et al (2016) Myeloperoxidase stimulates neutrophil degranulation. Bull Exp Biol Med 161(4):495-500

32. Chen KW et al (2014) The neutrophil NLRC4 inflammasome selectively promotes IL- $1 \beta$ maturation without pyroptosis during acute salmonella challenge. Cell Rep 8(2):570 
33. Sarkar A et al (2006) Caspase-1 regulates Escherichia coli sepsis and splenic B cell apoptosis independently of interleukin-1beta and interleukin-18. Am J Respir Crit Care Med 174(9):1003

34. Wang J et al (2017) Caspase-11 deficiency impairs neutrophil recruitment and bacterial clearance in the early stage of pulmonary Klebsiella pneumoniae infection. Int J Med Microbiol 307(8):490-496

35. Vanden BT et al (2014) Simultaneous targeting of IL-1 and IL-18 is required for protection against inflammatory and septic shock. Am J Respir Crit Care Med 18(2):1-53

36. Anasuya $\mathrm{S}$ et al (2006) Caspase-1 regulates Escherichia coli sepsis and splenic B cell apoptosis independently of interleukin-1beta and interleukin-18. Am J Respir Crit Care Med 174(9): 1003

37. Hotchkiss RS, Guillaume M, Didier P (2013) Sepsis-induced immunosuppression: from cellular dysfunctions to immunotherapy. Nat Rev Immunol 13(12):862-874

38. Kasten KR, Muenzer JT, Caldwell CC (2010) Neutrophils are significant producers of IL-10 during sepsis. Biochem Biophys Res Commun 393(1):28-31

39. Mcilwain DR, Berger T, Mak TW (2013) Caspase functions in cell death and disease. Cold Spring Harb Perspect Biol 5(4):a008656

40. Takeuchi O, Akira S (2010) Pattern recognition receptors and inflammation. Cell 140(6):805-820

41. Heil M, Land WG (2014) Danger signals-damaged-self recognition across the tree of life. Front Plant Sci 5(578):art. 578

42. Cullen SP et al (2015) Diverse activators of the NLRP3 inflammasome promote IL- $1 \beta$ secretion by triggering necrosis. Cell Rep 11(10):1535-1548

43. Chen G et al (2009) NOD-like receptors: role in innate immunity and inflammatory disease. Annu Rev Pathol 4(4):365

44. Schroder K, Tschopp J (2010) The inflammasomes. Cell 140(6):821

45. Lechtenberg BC, Mace PD, Riedl SJ (2014) Structural mechanisms in NLR inflammasome signaling. Curr Opin Struct Biol 29:17

46. Siyun K et al (2017) Structure insight of GSDMD reveals the basis of GSDMD autoinhibition in cell pyroptosis. PNAS 114(40):10642-10647

47. Xing L et al (2016) Inflammasome-activated gasdermin D causes pyroptosis by forming membrane pores. Nature 535(7610): 153

48. Shi J et al (2015) Cleavage of GSDMD by inflammatory caspases determines pyroptotic cell death. Nature 526(7575):660

49. Suzuki $T$ et al (2007) Differential regulation of caspase-1 activation, pyroptosis, and autophagy via Ipaf and ASC in shigellainfected macrophages. PLoS Pathog 3(8):e111

50. Sutterwala FS et al (2007) Immune recognition of Pseudomonas aeruginosa mediated by the IPAF/NLRC4 inflammasome. J Exp Med 204(13):3235

51. Zamboni DS (2006) The Birc1e cytosolic pattern-recognition receptor contributes to the detection and control of Legionella pneumophila infection. Nat Immunol 7(3):318

52. Roux CM et al (2007) Brucella requires a functional Type IV secretion system to elicit innate immune responses in mice. Cell Microbiol 9(7):1851-1869

53. Holzinger D et al (2012) Staphylococcus aureus Panton-Valentine leukocidin induces an inflammatory response in human phagocytes via the NLRP3 inflammasome. J Leukoc Biol 92(5): 1069

54. Greaney AJ, Leppla SH, Moayeri M (2015) Bacterial exotoxins and the inflammasome. Front Immunol 6(5):570

55. Craig L, Li J (2008) Type IV pili: paradoxes in form and function. Curr Opin Struct Biol 18(2):267-277
56. Martínsánchez F et al (2017) Lytic cell death induced by melittin bypasses pyroptosis but induces NLRP3 inflammasome activation and IL-1|[beta]| release. Cell Death Dis 8(8):e2984

57. Aglietti RA et al (2016) GsdmD p30 elicited by caspase-11 during pyroptosis forms pores in membranes. Proc Natl Acad Sci USA 113(28):7858-7863

58. Sborgi L et al (2016) GSDMD membrane pore formation constitutes the mechanism of pyroptotic cell death. EMBO J 35(16): 1766-1778

59. Ragland SA et al (2016) Two lytic transglycosylases in Neisseria gonorrhoeae impart resistance to killing by lysozyme and human neutrophils. Cell Microbiol 19:e12662

60. Ceballosolvera I et al (2011) Inflammasome-dependent pyroptosis and IL-18 protect against Burkholderia pseudomallei lung infection while IL-1 $\beta$ is deleterious. PLoS Pathog 7(12):e1002452

61. Monica M, Sabrina G, Carlo R (2016) The role of toll-like receptor 4 in infectious and noninfectious inflammation. Mediat Inflamm 2016(7):1-9

62. Wu Y et al (2015) The role of autophagy in kidney inflammatory injury via the NF- $\mathrm{KB}$ route induced by LPS. Int J Med Sci 12(8):655

63. Vanaja SK et al (2016) Bacterial outer membrane vesicles mediate cytosolic localization of LPS and caspase-11 activation. Cell 165(5): 1106

64. Kayagaki N et al (2011) Non-canonical inflammasome activation targets caspase-11. Nature 479(7371):117

65. Yang D et al (2015) Caspase-11 requires the pannexin-1 channel and the purinergic $\mathrm{P} 2 \mathrm{X} 7$ pore to mediate pyroptosis and endotoxic shock. Immunity 43(5):923

66. Välimäki E et al (2016) Calpain activity is essential for ATPdriven unconventional vesicle-mediated protein secretion and inflammasome activation in human macrophages. J Immunol 197(8):3315

67. Carlos TM et al (2016) Macrophage activation and polarization modify P2X7 receptor secretome influencing the inflammatory process. Sci Rep 6:22586

68. Hwang S-M et al (2009) P2X7 receptor-mediated membrane blebbing in salivary epithelial cells. Korean J Physiol Pharmacol 13(3): 175-179

69. Karmakar $\mathrm{M}$ et al (2015) Neutrophil IL-1 $\beta$ processing induced by pneumolysin is mediated by the NLRP3/ASC inflammasome and caspase- 1 activation and is dependent on $\mathrm{K}^{+}$efflux. $\mathrm{J}$ Immunol 194(4):1763-1775

70. Mausita K et al (2016) Neutrophil P2X7receptors mediate NLRP3 inflammasome-dependent IL- $1 \beta$ secretion in response to ATP. Nat Commun 7:10555

71. Surprenant A, North RA (2009) Signaling at purinergic P2X receptors. Annu Rev Physiol 71(71):333-359

72. Bartlett R, Stokes L, Sluyter R (2014) The P2X7 receptor channel: recent developments and the use of P2X7 antagonists in models of disease. Pharmacol Rev 66(3):638

73. Suh BC et al (2001) P2X7 nucleotide receptor mediation of membrane pore formation and superoxide generation in human promyelocytes and neutrophils. J Immunol 166(11):6754

74. Gu BJ et al (2000) Expression of $\mathrm{P} 2 \mathrm{X}_{7}$ purinoceptors on human lymphocytes and monocytes: evidence for nonfunctional $\mathrm{P}_{2} \mathrm{X}_{7}$ receptors. Am J Physiol Cell Physiol 279(4):C1189-C1197

75. Wang $X$ et al (2017) Endotoxin-induced autocrine ATP signaling inhibits neutrophil chemotaxis through enhancing myosin light chain phosphorylation. Proc Natl Acad Sci USA 114(17):4483

76. Levin R, Grinstein S, Canton J (2016) The life cycle of phagosomes: formation, maturation, and resolution. Immunol Rev 273(1):156 
77. Dubois H, Wullaert A, Lamkanfi M (2016) General strategies in inflammasome biology. Springer International Publishing, Switzerland

78. Criss AK, Seifert HS (2012) A bacterial siren song: intimate interactions between neutrophils and pathogenic Neisseria. Nat Rev Microbiol 10(3):178-190

79. Guerra FE et al (2017) Epic immune battles of history: neutrophils vs. Staphylococcus aureus. Front Cell Infect Microbiol 7:286

80. Gieffers J et al (2004) Phagocytes transmit Chlamydia pneumoniae from the lungs to the vasculature. Eur Respir $\mathbf{J}$ 23(4):506-510

81. Ge Y et al (2005) Anaplasma phagocytophilum inhibits human neutrophil apoptosis via upregulation of bfl-1, maintenance of mitochondrial membrane potential and prevention of caspase 3 activation. Cell Microbiol 7(1):29-38

82. Arnett $\mathrm{E}$ et al (2014) The pore-forming toxin listeriolysin $\mathrm{O}$ is degraded by neutrophil metalloproteinase- 8 and fails to mediate Listeria monocytogenes intracellular survival in neutrophils. $\mathbf{J}$ Immunol 192(1):234

83. Yang J, Zhao Y, Shao F (2015) Non-canonical activation of inflammatory caspases by cytosolic LPS in innate immunity. Curr Opin Immunol 32:78

84. Boyce AK et al (2014) Pore positioning: current concepts in pannexin channel trafficking. Channels 8(2):110-117

85. Taylor KA, Wright JR, Mahaut-Smith MP (2015) Regulation of pannexin-1 channel activity. Biochem Soc Trans 43(3):502

86. Shi J et al (2014) Inflammatory caspases are innate immune receptors for intracellular LPS. Nature 514(7521):187-192

87. Kayagaki $\mathrm{N}$ et al (2013) Noncanonical inflammasome activation by intracellular LPS independent of TLR4. Science 341(6151):1246-1249

88. Kayagaki $\mathrm{N}$ et al (2015) Caspase-11 cleaves gasdermin D for non-canonical inflammasome signalling. Nature 526(7575):666

89. Baldauf KJ et al (2015) Cholera toxin B: one subunit with many pharmaceutical applications. Toxins 7(3):974

90. Jan AT (2017) Outer membrane vesicles (OMVs) of gram-negative bacteria: a perspective update. Front Microbiol 8:1053

91. Dorward DW, Garon CF (1990) DNA is packaged within membrane-derived vesicles of gram-negative but not gram-positive bacteria. Appl Environ Microbiol 56(6):1960-1962

92. Deng M et al (2018) The endotoxin delivery protein HMGB1 mediates caspase-11-dependent lethality in sepsis. Immunity 49(4):740-753.e7

93. Krumm B, Xiang Y, Deng J (2014) Structural biology of the IL-1 superfamily: key cytokines in the regulation of immune and inflammatory responses. Protein Sci 23(5):526-538

94. Dinarello CA (2009) Immunological and inflammatory functions of the interleukin-1 family. Annu Rev Immunol 27(27):519

95. Bergsbaken T, Fink SL, Cookson BT (2009) Pyroptosis: host cell death and inflammation. Nat Rev Microbiol 7(2):99

96. Sedimbi SK, Hägglöf T, Karlsson MCI (2013) IL-18 in inflammatory and autoimmune disease. Cell Mol Life Sci 70(24):4795-4808

97. Tsutsui H, Nishiguchi S (2014) Importance of Kupffer cells in the development of acute liver injuries in mice. Int J Mol Sci 15(5):7711-7730

98. Pillon NJ et al (2016) Saturated fatty acids activate caspase-4/5 in human monocytes, triggering IL- $1 \beta$ and IL-18 release. Am J Physiol Endocrinol Metab 311(5):E825-E835

99. Veerdonk FLVD, Netea MG (2011) Inflammasome activation and IL-1 $\beta$ and IL-18 processing during infection. Trends Immunol 32(3): 110

100. Sahoo $\mathrm{M}$ et al (2011) Role of the inflammasome, IL-1 $\beta$, and IL-18 in bacterial infections. Sci World J 11:2037
101. Andrei C et al (2004) Phospholipases C and A2 control lysosome-mediated IL-1 $\beta$ secretion: implications for inflammatory processes. Proc Natl Acad Sci USA 101(26):9745-9750

102. Brough D, Rothwell NJ (2007) Caspase-1-dependent processing of pro-interleukin- $1 \beta$ is cytosolic and precedes cell death. J Cell Sci 120(5):772-781

103. Qu Y et al (2007) Nonclassical IL-1 beta secretion stimulated by $\mathrm{P} 2 \mathrm{X} 7$ receptors is dependent on inflammasome activation and correlated with exosome release in murine macrophages. J Immunol 179(3):1913-1925

104. Pizzirani C et al (2007) Stimulation of P2 receptors causes release of IL-1 $\beta$-loaded microvesicles from human dendritic cells. Blood 109(9):3856-3864

105. Bianco F et al (2005) Astrocyte-derived ATP induces vesicle shedding and IL-1 beta release from microglia. J Immunol 174(11):7268-7277

106. MacKenzie A et al (2001) Rapid secretion of interleukin- $1 \beta$ by microvesicle shedding. Immunity 15(5):825-835

107. Evavold CL et al (2018) The pore-forming protein gasdermin $\mathrm{D}$ regulates interleukin-1 secretion from living macrophages. Immunity 48(1):35-44

108. Kaplan MJ, Radic M (2012) Neutrophil extracellular traps: double-edged swords of innate immunity. J Immunol 189(6):2689-2695

109. Remijsen Q et al (2011) Dying for a cause: NETosis, mechanisms behind an antimicrobial cell death modality. Cell Death Differ 18(4):581-588

110. Braian C, Hogea V, Stendahl O (2013) Mycobacterium tuberculosis- induced neutrophil extracellular traps activate human macrophages. J Innate Immun 5(6):591-602

111. Stephan A, Fabri M (2015) The NET, the trap and the pathogen: neutrophil extracellular traps in cutaneous immunity. Exp Dermatol 24(3):161-166

112. Remijsen Q et al (2011) Neutrophil extracellular trap cell death requires both autophagy and superoxide generation. Cell Res 21(2):290

113. Poon IKH, Hulett MD, Parish CR (2010) Molecular mechanisms of late apoptoticl[sol] Inecrotic cell clearance. Cell Death Differ 17(3):381

114. Peter C et al (2010) Dangerous attraction: phagocyte recruitment and danger signals of apoptotic and necrotic cells. Apoptosis Int J Program Cell Death 15(9):1007-1028

115. Labbé K, Saleh M (2008) Cell death in the host response to infection. Cell Death Differ 15(9):1339

116. Marshall JC (2014) Why have clinical trials in sepsis failed? Trends Mol Med 20(4):195-203

117. Tom VB et al (2013) An inactivating caspase-11 passenger mutation muddles sepsis research. Am J Respir Crit Care Med 188(1): 120

118. Gentile LF et al (2015) Improved emergency myelopoiesis and survival in neonatal sepsis by caspase-1/11 ablation. Immunology $145(2): 300-311$

119. Lee $\mathrm{S}$ et al (2017) NLRP3 inflammasome deficiency protects against microbial sepsis via increased lipoxin B4 synthesis. Am J Respir Crit Care Med 196(6):713

120. Sanjeev M et al (2004) Differential activation of the inflammasome by caspase-1 adaptors ASC and Ipaf. Nature 430(6996):213-218

121. Fisher CJ et al (1994) Recombinant human interleukin 1 receptor antagonist in the treatment of patients with sepsis syndrome: results from a randomized, double-blind, placebo-controlled trial. JAMA 271(23):1836-1843

122. Opal SM et al (1997) Confirmatory interleukin-1 receptor antagonist trial in severe sepsis: a phase III, randomized, double-blind, placebo-controlled, multicenter trial. The Interleukin- 1 
Receptor Antagonist Sepsis Investigator Group. Crit Care Med 25(7):1115-1124

123. Shakoory B et al (2015) Interleukin-1 receptor blockade is associated with reduced mortality in sepsis patients with features of macrophage activation syndrome: reanalysis of a prior phase III trial. Crit Care Med 44(2):275

124. Meyer NJ et al (2018) Mortality benefit of recombinant human interleukin-1 receptor antagonist for sepsis varies by initial interleukin-1 receptor antagonist plasma concentration. Crit Care Med 46(1):21-28

125. Surender R et al (2014) Therapeutic role of anakinra, an interleukin-1 receptor antagonist, in the management of secondary hemophagocytic lymphohistiocytosis/sepsis/multiple organ dysfunction/macrophage activating syndrome in critically ill children. Pediatr Crit Care Med 15(5):401-408
126. Singh N et al (2017) Inflammasome expression and cytomegalovirus viremia in critically ill patients with sepsis. J Clin Virol 93:8-14

127. Wang YC et al (2018) Caspase-1-dependent pyroptosis of peripheral blood mononuclear cells predicts the development of sepsis in severe trauma patients: a prospective observational study. Medicine (Baltimore) 97(8):e9859

Publisher's Note Springer Nature remains neutral with regard to jurisdictional claims in published maps and institutional affiliations. 